\title{
PERFIL ANTROPOMÉTRICO E COMPOSIÇÃO CORPORAL DE MULHERES PRATICANTES DE CORRIDA DE RUA
}

DOI: 10.48140/digitaleditora.2020.001.6

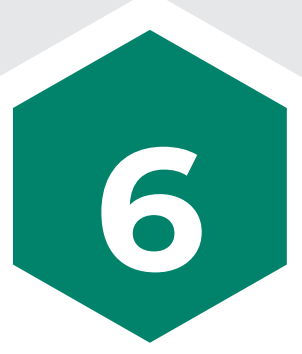

RESUMO

Objetivos: Desta maneira, o presente estudo tem como objetivo investigar por meio de uma pesquisa narrativa. Analisar e avaliar perfil antropométrico e composição corporal de mulheres praticantes de corrida de rua.

Métodos: Trata-se de um estudo de caráter qualitativo realizado por meio de pesquisa bibliográfica, constituído de artigos científicos. A coleta de dados sobre perfil antropométrico e composição corporal em mulheres corredoras de rua que será realizado a partir de artigos científicos nas principais bases de dados científicos, tais como Google acadêmico, BIREME (Centro Latino-Americano e do Caribe de Informação em Ciências da Saúde) PubMed, RBPFEX (revista brasileira de prescrição e fisiologia do exercício) e Scielo, O estudo foi realizado considerando buscando apresentar a importância sobre o assunto, através de alguns autores, desde o ano de 2002 a 2020. Resultados: Consta-se que o uso das variáveis corporais como a antropometria e a composição corporal é indispensável para a realização de um treinamento, pois cada indivíduo tem sua individualidade biológica, para isso é necessário avaliar esses parâmetros.

Conclusão: Portanto relata-se a importância das variáveis perfil antropométrico e composição corporal nos indivíduos para realizar o treino individualizado na busca dos objetivos desejados.
Ana Paula de Sousa Martins

Graduanda em Educação Física pela AESPI - Ensino Superior do Piauí

Teresina - Piauí

iD https://orcid.org/0000.

iD 0003-1480-2461

Antonia Rita de Cássia Oliveira Graduanda em Educação Física pela AESPI - Ensino Superior do Piauí

Teresina - Piauí

iD

https://orcid.org/0000 0003-0254-8849

\section{Rômulo Matos Pinheiro}

Profissional de Educação Física, Mestre e Professor Assistente da AESPI - Ensino Superior do Piauí Teresina - Piauí

iD https://orcid.org/0000 0002-9812-8170 


\section{ANTHROPOMETRIC PROFILE AND BODY COMPOSITION OF WOMEN PRACTICING STREET RACING}

DOI: $10.48140 /$ digitaleditora.2020.002.6

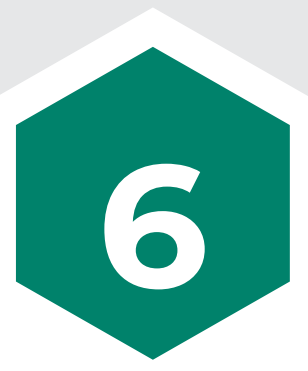

ABSTRACT

Recebido em: 10/12/2020

Aprovado em: 28/12/2020

Conflito de Interesse: não

Suporte Financeiro: não houve

Objectives: In this way, the present study aims to investigate through narrative research analyze and evaluate anthropometric profile and body composition of women who practice street running.

Methods: This is a qualitative study carried out through bibligraphic research, consisting of scientific articles. The collection of data on anthopometric profile and body composition in female street runners that will be carried out from scientific articles in the main scientific databases, such as google acadêmic, BIREME( Latin American and Caribbean center for health Sciences information) pubMed, RBPFEX( Brazilian journal of prescription and exercise physiology) ande Scielo, The study was carried out considering the importance of the subject, through some authours, from 2002 to 2020.

Results: It appears that the use of body variables such as anthropometry and body composition is essential for training, since each individual has his or her biology individuality, for this it is necessary to evaluate these parameters

Conclusion: Therefore, it is reported the importance of the variables anthropometric profile and body composition in which we are obliged to carry out individualized training in search of the desired objectives. 


\section{INTRODUÇÃO}

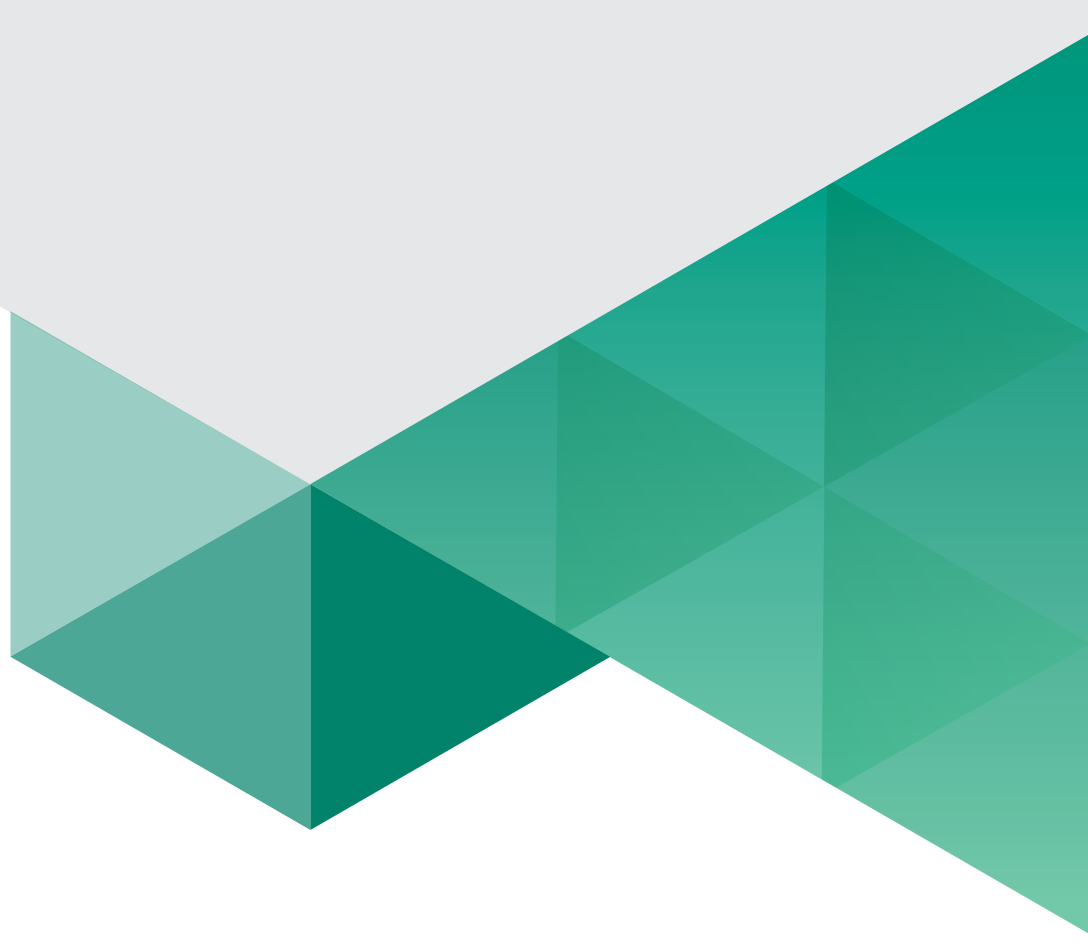

A corrida de rua se apresenta como uma das modalidades esportivas, com uma grande quantidade de simpatizantes. Tal interesse reside no fato de sua prática promover a melhora da qualidade de vida de seus adeptos, sem que para isso estes precisem investir altos montantes (LOPES, SARAGIOTTO E YAMATO, 2011).

Estudos sobre o perfil antropométrico e composição, detêm importância fundamental, a partir dele as mulheres praticante de corrida, pôde diversificar sobres os treinos ou melhor personaliza-los. É fundamental o acompanhamento da composição corporal das atletas, sendo um meio importante para o controle dos treinamentos (PECARARO E GRECO 2006), no estabelecimento das necessidades nutricionais, peso e percentual de gordura adequados, baseado nos aspectos fisiológicos, antropométricos e dietéticos (SBME, 2003; HICKSON JUNIOR, 2002).

O conhecimento do perfil antropométrico trata das medidas físicas do corpo humano. Conforme Petroski (2007), estudos sobre o perfil antropométrico detém importância fundamental, com as características físicas dos homens assim como as medições e avaliações de diferentes aspectos do mesmo, em movimento. Partir delas se pôde diversificar e complementar estudos através da mesma. Os estudos da composição corporal são possíveis a partir de medidas antropométricas, tais medidas podem avaliar o estado e risco de obesidade, tanto de crianças, jovens, adultos e idosos.

Hoje o estudo da antropometria não está apenas voltado para ser um indicador de saúde, está sendo muito utilizado para fins de aprimorar o desempenho esportivo de atletas e não atletas, melhora na performance, embasado em corredores que precisam de uma alta performance e para avaliar o estado físico e de saúde dos alunos. Atualmente, a técnica antropométrica é um dos procedimentos de maior aplicabilidade para avaliação nutricional de indivíduos, atletas ou não, em virtude do custo e aceitabilidade do método (GUEDES E GUEDES, 2003).

$\mathrm{Na}$ área da Educação Física os dados antropométricos, enquanto avaliação da composição corporal tem um importante papel em fornecer respostas necessárias à elaboração de um correto progra- 
ma de atividade física. Em outras palavras, para que uma atividade física atinja o objetivo pretendido, é necessário que a carga de trabalho motor seja individualizada para cada atleta. E para que seja benéfica ao praticante, faz-se necessária uma avaliação física voltada ao objetivo individual, com variáveis que forneça dados importantes na elaboração e reajuste dos programas de atividade física.

A avaliação da composição corporal realizada para qualificar e fornecer dados relativos à quantidade dos principais componentes estruturais do corpo humano: musculaturas, ossos, gorduras corporais, tecidos e substâncias residuais. Esta técnica utiliza de variáveis como dobras cutâneas, perímetros, idade, peso e estatura, elas sozinhas ou combinadas, são usadas para obtenção de índices, tais como o índice de massa corporal (IMC), ou o percentual de gordura (\%G) (PECARARO GRECO, 2006; GLANER, 2005).

O método antropométrico alternativo para análise da composição corporal consiste nas medidas de perímetros em regiões específicas do corpo. Para a realização das medidas de perímetros é empregada fita antropométrica flexível que permita aplicar pressão constante sobre a superfície da pele durante toda a medição. A antropometria apresenta informações valiosas para a predição e a estimação dos vários componentes corporais de sedentarismo ou atletas no crescimento, desenvolvimento e envelhecimento (FILHO, 2003). E fundamental sabermos qual o tipo o biótipo físico se enquadrar de tal forma e possível traçar diretrizes, para atingir excelentes resultados no esporte praticado.

O objetivo geral foi avaliar perfil antropométrico e composição corporal de mulheres praticantes de corrida de rua, visto que os objetivos específicos: Verificar a composição corporal das mulheres praticante da corrida, caracterizar o perfil antropométrico das mulheres praticantes da corrida, identificar alterações na composição corporal nas mulheres praticante de corrida de rua.

Analisar o perfil antropométrico e composição corporal de mulheres praticantes de corrida de rua, apesar dos corredores ter um básico conhecimento em técnicas de corrida muitos procuram um profissional de educação física, visto que também o acompanhamento com a nutricionista e indispensável para a obtenção de resultados.

Portanto a proposta do projeto e averiguar os perfis antropométricos e composições corporais nas mulheres praticantes de corrida pois a corrida serve para muitos como uma forma de resgatar e despertar um estilo de vida ativa, por ser de fácil acesso acabou se tornando uma boa alternativa, além de demonstra aumento dos benefícios à saúde e qualidade de vida relacionado ao aumento do gasto energético na execução da corrida. 


\section{METODOLOGIA TIPOS DE ESTUDO}

O presente estudo trata-se de uma revisão bibliográfica do tipo narrativa, que foi realizada por meio de um levantamento de literatura onde foram utilizadas fontes secundárias referentes à temática em questão, no qual abordou sobre o perfil antropométrico e composição corporal em mulheres corredoras de rua, A pesquisa bibliográfica é elaborada com base em material já publicado a modalidade de pesquisa inclui estudos encontrados nas bases de dados do Google acadêmico, BIREME (Centro Latino-Americano e do Caribe de Informação em Ciências da Saúde), Scielo, RBPFEX (revista brasileira de prescrição e fisiologia do exercício) e pubMed, datados entre 2002 a 2020. Tivemos também como base artigos científicos, trabalho de conclusão de graduação e teses de mestrados em língua portuguesa.

De acordo com Rother (2007) os artigos de revisão narrativa são textos que constituem a análise da literatura científica na interpretação e análise crítica do autor. A despeito de sua força de evidência científica ser considerada baixa devido à impossibilidade de reprodução de sua metodologia, as revisões narrativas podem contribuir no debate de determinadas temáticas, levantando questões e colaborando na aquisição e atualização do conhecimento em curto espaço de tempo.

\section{CRITÉRIOS DE INCLUSÃO E EXCLUSÃO}

Estudos que analisaram a composição corporal, perfil antropométrico; Artigos em português, com texto completo disponível nas bases de dados no período compreendido entre 2002 e 2020. Exclusão os estudos que não analisaram composição corporal, perfil antropométrico, o texto completo disponível ou fora do período entre 2002 a 2020.

\section{PROCEDIMENTOS PARA COLETAS DE DADOS}

Para a realização desta pesquisa foi feito um levantamento bibliográfico para seleção dos estudos nas bases de dados on-line: The Scientific Electronic Library Online - (SCIELO), e pubMed, onde selecionou alguns artigos por meio da associação dos seguintes descritores

1ㅇassociação: perfil antropométrico e composição corporal.

2o associação: perfil corporal e corrida de rua. 
3o associação: composição corporal e estrutura corporal e perfil antropométrico

\section{ANÁLISES DOS DADOS}

Finalizada a coleta de dados, foi realizada uma análise dos objetivos e resultados de todos os estudos a fim de obter os pontos de partida e desfechos que mais fundamentaram o tema pesquisado. Em seguida foi feita a leitura de todo o material escolhido e reunido as principais informações que buscaram estabelecer uma compreensão e ampliar o conhecimento sobre o tema estudado para que seja elaborado o referencial teórico.

\section{RESULTADOS}

Nesta revisão narrativa foram incluídos (27) artigos nas bases de dados SCIELO (Scientific Eletronic Library On line), BIREME (Centro Latino-Americano e do Caribe de Informação em Ciências da Saúde) e PubMed. Os artigos apresentados em mais de uma base de dados foram contabilizados apenas uma vez (Figura 1).

FIGURA 1. Quantidade de artigos conforme a base de dados

\begin{tabular}{|c|c|c|c|}
\hline \multicolumn{4}{|c|}{ Após utilizar os critérios de inclusão foram encontrados: } \\
\hline $\begin{array}{c}08 \text { artigos da } \\
\text { BIREME }\end{array}$ & $\begin{array}{l}03 \text { artigos da } \\
\text { RBPFEX }\end{array}$ & $\begin{array}{l}10 \text { artigos da } \\
\text { SCIELO }\end{array}$ & $\begin{array}{c}06 \text { artigos do PUB } \\
\text { MED }\end{array}$ \\
\hline & $\downarrow$ & $\downarrow$ & $\downarrow_{02}$ \\
\hline $\begin{array}{l}\text { Deste } 01 \text { artigo } \\
\text { atendeu a questão } \\
\text { da pesquisa }\end{array}$ & $\begin{array}{lr}\text { Destes } & 02 \\
\text { atendeu } & \text { artigos } \\
\text { questão } & \text { da }\end{array}$ & $\begin{array}{lr}\text { Destes } 03 & \text { artigos } \\
\text { atendeu } & \mathrm{a} \\
\text { questão } & \text { da }\end{array}$ & $\begin{array}{ll} & \text { Destes } 02 \\
\text { atenderam } & \text { artigos } \\
\text { questão } & \text { da } \\
\text { pesquisa } & \end{array}$ \\
\hline
\end{tabular}

Fonte: Autoria Própria 2020 
Quadro 01. Distribuição dos autores, base de dados, objetivos e resultados.

\begin{tabular}{|c|c|c|c|}
\hline $\begin{array}{l}\text { AUTORES I } \\
\text { ANO }\end{array}$ & $\begin{array}{l}\text { BASE DE } \\
\text { DADOS }\end{array}$ & OBJETIVO DE ESTUDO & RESULTADOS \\
\hline $\begin{array}{l}\text { Dartagnan } \\
\text { et.al (2008). }\end{array}$ & Bireme & $\begin{array}{l}\text { Determinar a concordância } \\
\text { entre os valores de gordu- } \\
\text { ra corporal estimados por } \\
\text { quatro equações envolven- } \\
\text { do medidas de espessura de } \\
\text { dobras cutâneas e equação, } \\
\text { envolvendo o índice de massa } \\
\text { corporal (IMC) em estudantes } \\
\text { universitários. }\end{array}$ & $\begin{array}{l}\text { Os sujeitos reunidos no estudo apresentaram ida- } \\
\text { des entre } 18 \text { e } 30 \text { anos e, quando da comparação } \\
\text { entre os valores médios observados em ambos os } \\
\text { sexos, as diferenças encontradas não são apontadas } \\
\text { em linguagem estatística }(p=0,1170) \text {. }\end{array}$ \\
\hline $\begin{array}{l}\text { Nayza et.al } \\
\text { ( 2017). }\end{array}$ & RBPFEX & $\begin{array}{l}\text { Verificar a concepção da ima- } \\
\text { gem corporal de um grupo de } \\
\text { praticantes de corrida de rua, } \\
\text { realizando uma correlação } \\
\text { com a prática regular de ati- } \\
\text { vidade física, e consequente- } \\
\text { mente, com condicionamento } \\
\text { físico do público alvo. }\end{array}$ & $\begin{array}{l}\text { Pode-se apontar que } 80 \text { entrevistados ( } \pm 73 \% \\
\text { da amostra), isto é, } 39 \text { mulheres }( \pm 49 \% \text { ) e } 41 \\
\text { homens }( \pm 51 \%) \text {, desejam diminuir gordura } \\
\text { corporal, estando insatisfeitos corporalmente. } \\
\text { Enquanto, } 13 \text { participantes ( } \pm 12 \% \text { da amos- } \\
\text { tra), } 5 \text { mulheres }( \pm 38 \% \text { ) e } 8 \text { homens ( } 62 \% \text { ) } \\
\text { disseram que estão satisfeitos corporalmente. } \\
\text { E por fim, } 9 \text { entrevistados ( } \pm 8 \% \text { da amostra), } 2 \\
\text { mulheres ( } \pm 22 \% \text { e } 7 \text { homens ( } \pm 78 \% \text { ), relata- } \\
\text { ram que desejam ganhar massa magra, apre- } \\
\text { sentando também a insatisfação com o corpo } \\
\text { atual. }\end{array}$ \\
\hline $\begin{array}{l}\text { Charline et.al } \\
\text { (2012). }\end{array}$ & Scielo & $\begin{array}{l}\text { Examinar o perfil antropomé- } \\
\text { trico, o consumo alimentar e } \\
\text { conhecimento nutricional de } \\
\text { corredores de rua. }\end{array}$ & $\begin{array}{l}\text { Os resultados mostraram IMC, relação cintu- } \\
\text { ra-quadril e percentual de gordura adequado, } \\
\text { bom conhecimento nutricional, porém ina- } \\
\text { dequações dietéticas na ingestão de calorias, } \\
\text { percentual de carboidratos da dieta e cálcio, } \\
\text { embora } 40 \% \text { dos avaliados já tenham passado } \\
\text { por orientação nutricional. }\end{array}$ \\
\hline $\begin{array}{l}\text { Gustavo Wa- } \\
\text { clawovsky } \\
\text { et.al (2018). }\end{array}$ & pubMed & $\begin{array}{l}\text { Analisar a relação entre compo- } \\
\text { sição corporal, aspectos nutricio- } \\
\text { nais e gênero com o VO2máx de } \\
\text { corredores de longa distância. }\end{array}$ & $\begin{array}{l}\text { O VO2máx foi menor e o \%G foi maior no gru- } \\
\text { po feminino versus o masculino ( } p<0,001 \text { para } \\
\text { ambas comparações). O VO2máx correlacio- } \\
\text { nou-se inversamente com o \%G ( } r=-0,655 \text {; } \\
p<0,001 \text { ) com o somatório de sete dobras } \\
\text { cutâneas ( } r=-0,599 ; p=0,001) \text {. }\end{array}$ \\
\hline $\begin{array}{l}\text { Vanessa et.al } \\
\text { (2015). }\end{array}$ & Scielo & $\begin{array}{l}\text { Avaliar a adequação dietética, } \\
\text { o perfil antropométrico e os } \\
\text { conhecimentos em nutrição } \\
\text { de corredores de rua. }\end{array}$ & $\begin{array}{l}\text { A maioria dos corredores de rua do gênero } \\
\text { masculino teve um percentual de gordura ade- } \\
\text { quado e foi constatada magreza para cerca de } \\
30 \% \text { de homens e mulheres. As inadequações } \\
\text { dietéticas de macro nutrientes caracterizaram- } \\
\text {-se por consumo insuficiente de carboidratos } \\
\text { ( } 59,6 \% \text { de indivíduos) e ingestão elevada de } \\
\text { proteínas ( } 32,7 \%) \text {. Fibra, cálcio, tiamina, ribo- } \\
\text { flavina e niacina foram consumidas abaixo dos } \\
\text { níveis recomendados, principalmente entre os } \\
\text { homens. }\end{array}$ \\
\hline
\end{tabular}




\begin{tabular}{|c|c|c|c|}
\hline $\begin{array}{l}\text { Edvan et.al } \\
\text { (2016) }\end{array}$ & RBPFEX & $\begin{array}{l}\text { Caracterizar o perfil antropo- } \\
\text { métrico e de ingesta dietética } \\
\text { de corredores de rua do sexo } \\
\text { masculino e feminino de uma } \\
\text { assessoria de corrida da cida- } \\
\text { de de Curitiba-PR. }\end{array}$ & $\begin{array}{l}\text { Foi verificado, que os atletas masculinos } 77,8 \% \\
\text { possuíam perfil hipoglicídico, } 88,9 \% \text { eram hi- } \\
\text { perproteico, as atletas femininas 100\% eram } \\
\text { hipoglicídica e hiperproteica e } 83,3 \% \text { estavam } \\
\text { hiperlipídicas. Com os dados da presente pes- } \\
\text { quisa, pode ser concluído que tanto os corre- } \\
\text { dores homens como mulheres apresentam } \\
\text { inadequação em suas dietas alimentares. }\end{array}$ \\
\hline $\begin{array}{l}\text { Roselaine } \\
\text { et.al (2010) }\end{array}$ & Scielo & $\begin{array}{l}\text { Descrever o perfil antropomé- } \\
\text { trico de mulheres participan- } \\
\text { tes de um grupo de corrida de } \\
\text { rua do município de campo } \\
\text { Grande-MS. }\end{array}$ & $\begin{array}{l}\text { De acordo com o IMC, o grupo estudado apre- } \\
\text { sentou } 82,5 \% \text { classificado como normal, } 2,5 \% \\
\text { com o peso baixo e } 15,0 \% \text { com peso corporal } \\
\text { elevado. Ao analisar o percentual de gordura, } \\
60 \% \text { da população obtiveram percentuais ex- } \\
\text { celentes, } 10 \% \text { com classificação boa, } 27,5 \% \\
\text { exato na média e } 2,5 \% \text { muito elevado. Com os } \\
\text { resultados descritos, entende-se que as atletas } \\
\text { de corrida de rua apresentam normalidade no } \\
\text { que se refere aos índices antropométrico, pois } \\
\text { grande parte do grupo obteve resultados satis- } \\
\text { fatório em relação a dados normativos para a } \\
\text { saúde. }\end{array}$ \\
\hline $\begin{array}{l}\text { Demétrius } \\
\text { et.al (2012) }\end{array}$ & pubMed & $\begin{array}{l}\text { Traçar o perfil de gordura dos } \\
\text { praticantes de caminhada/ } \\
\text { corrida em praças públicas, } \\
\text { com a faixa etária dos } 18 \text { aos } \\
90 \text { anos, independentemente } \\
\text { do sexo }\end{array}$ & $\begin{array}{l}\text { Conclui-se que o estudo demonstrou prevalên- } \\
\text { cia de obesidade leve e sobrepeso nos prati- } \\
\text { cantes de caminhada e corrida, encontrando- } \\
\text {-se um fator risco a saúde muito aumentado. } \\
\text { Comparado com o IRCQ, encontrou-se risco } \\
\text { muito alto. }\end{array}$ \\
\hline
\end{tabular}

Fonte: Pesquisa realizada em bases de dados.

\section{DISCUSSÃO}

Podemos perceber eventual concordância entre os valores de gordura corporal estimados por equações envolvendo medidas de espessura das dobras cutâneas e valores equivalentes ao IMC foi analisada mediante três procedimentos estatísticos: teste " $t$ " de Student para dados pareados, coeficiente de correlação intraclasse e plotagem em diagrama de dispersão de acordo com a técnica desenvolvida por (BLAND E ALTMAN). O IMC não é considerado o indicador mais adequado para indivíduos fisicamente ativos, visto que não considera os componentes da massa corporal, porem a importância da composição corporal, pois as características antropométricas de homens e mulheres podem ter associações diferentes com o desempenho da corrida.

Esses estudos são consistentes com informações provenientes de estudo disponibilizado na literatura envolvendo valores de \%Gordura estimados pelo Modelo IMC e por outros modelos antropométricos com participação de medidas equivalentes à espessura de dobras cutâneas, ver-se ainda a influência da composição corporal, em especial o percentual de gordura, sobre o VO2máx de praticantes de corrida de rua.

O percentual de insatisfação (diminuir gordura e aumentar massa magra) e satisfação corporal foi maior no público masculino em comparação ao feminino, o que difere aos estudos, que dizem que quando comparado ao sexo, às mulheres são mais insatisfeitas corporalmente, por muitas vezes 
não gostar de academia ambiente fechado, por questão de custos as pessoas começam a fazer corrida no intuito de melhorar a qualidade vida assim se encontrando no esporte como resultados encontrados na pesquisa de KAKESHITA E ALMEIDA (2006).

Dados obtidos por esta revisão apresentam uma amostra de corredores jovens, com composição corporal adequada para idade, ingestão dietética abaixo de algumas recomendações e bom conhecimento nutricional de acordo com os percentuais de acerto. Os percentuais de gordura corporal encontrados refletem positivamente na adaptação ao treinamento de longa distância e facilitam a perda de calor para o meio. (McArdle, Katch e Katch, 2003). Além disso, uma baixa carga de peso corporal também auxilia, indiretamente, no rendimento por diminuir a sobrecarga imposta às articulações (NEWSHOLME E COLABORADORES, 1994).

A composição corporal é uma variável que oferece informações úteis para medir o estado de saúde e nutricional de um indivíduo, bem como servir de indicador do desempenho em várias modalidades esportivas (GOSTON E MENDES, 2011).

Portanto, é fundamental importância A investigação da variável gordura corporal, pois indivíduos com níveis elevados deste componente corporal, aumentam nas chances de desenvolver várias doenças, como diabetes, hipertensão arterial, dislipidemias e doenças cardiovasculares, além disso a periodização e essencial para os atletas conforme Wilmore e Costill (2001) afirmam que cada modalidade esportiva tem uma composição corporal ideal, e com relação à corrida de rua, quanto menor a massa gorda, melhor o desempenho na prova. 


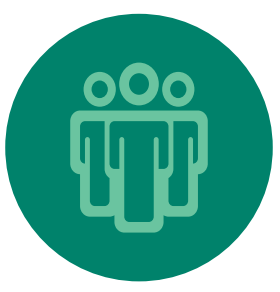

\section{CONCLUSÃO}

Os resultados vistos no estudo relatam que os que o uso da avaliação antropométrica e composição corporal são ferramentas que não só auxiliam, como também são de suma importância para o treinamento e busca de resultados na pratica de corrida de rua pois, estar interligados para determinar o rendimento do atleta de endurance. Desta forma corrobora a importância da participação do profissional de educação física para obter os resultados desejados e específicos para cada pessoa tem-se a individualidade biológica. Por ser variáveis bastantes precisas a antropometria e a composição corporal e útil na avaliação de praticantes de exercício físico pois, nelas mostram as devidas proporções corporais de água, ossos músculos e gordura, bem como uma estimativa de massa magra e massa gorda, que está diretamente ligado na amostra de resultados, então sugere-se a utilização de outros testes complementares para a boa prescrição do exercício físico orientado. 


\section{REFERÊNCIAS}

ABERNETHY, Peter; OLDS, Tim; EDEM, Edem; NEILL, Michelle \& BAINESS, Linda. Antropometria, Saúde e Composição corporal. In NORTON, Kevin \& OLDS, TIM. Antropométrica. Editora Artmed, 2005.

BLAND JM, Altman DG. Statistical methods for assessing agreement between two methods of clinical measurement. Lancet 1986;

FERNANDES FILHO, JOSÉ. A prática da avaliação física, 2o ed Rio de Janeiro: Shape, 2003.

GLANER, M.F. Índice de massa corporal como indicativo da gordura corporal comparado às dobras cutâneas. Revista Brasileira de Medicina do Esporte. Vol. 11. Num. 4. 2005.

GOSTON, J. L.; MENDES, L. L. Perfil Nutricional de Praticantes de Corrida de Rua de Um Clube Esportivo da Cidade de Belo Horizonte, MG, Brasil.Rev. Bras. Med. Esporte. Vol. 17. Num. 1. 2011. p.13-17.

GUEDES, DARTAGNAN PINTO \& GUEDES, Joana Elisabete Ribeiro. Controle do peso corporal: composição corporal, atividade física e nutrição. 2. ed. Rio de Janeiro: Shape, 2003.

HEYWARD, V. H.; STOLARCZYK, L. M. Avaliação da composição corporal aplicada. Editora Manole Ltda.: São Paulo, 2000.

HICKSON JUNIOR, J.F. Nutrição no Exercício e no esporte. São Paulo. Roca. 2002.

-KAKESHITA, I. S.; ALMEIDA, S.S. Relação entre índice de massa corporal e a percepção da auto-imagem em universitários. Revista de Saúde Pública. Vol. 40. Num. 3. 2006. p. 497- 504.

MCARDLE, W. D; KATCH, F. I.; KATCH, V. L. Fisiologia do exercício; energia, nutrição. 5. ed., Guanabara Koogan, 2003.

NEWSHOLME, E.A.; LEECH, T.; DUESTER, G. Keep on running: the science of training and performance. Chichester, John Willey, 1994. p.50-157.

PAZIN, J.; DUARTE, M. D. S.; POETA, L. S.; GOMES, M. A. Corredores de rua: características demográficas, treinamento e prevalência de lesões. Revista Brasileira de Cineantropometria e Desempenho Humano, v. 10, n 3, 2008.

PECARARO, S.P.; GRECO, C.C. Comparação de diferentes equações propostas para a estimativa da densidade e do percentual de gordura corporal. Revista do Centro Universitário Claretiano. Batatais. Vol. 1. 2006. p. 174-182.

PETROSKI, Edio Luiz. Antropometria: técnicas e padronizações. 3 ed. Nova Letra, Blumenau, SC, 1999; 
ROTHER ET. Revisão sistemática X revisão narrativa. Acta paul. Enferm 2007; 20(2):vvi.

Sociedade Brasileira de Medicina do Esporte (SBME). Diretriz: Modificações dietéticas, reposição hídrica, suplementos alimentares e drogas: comprovação de ação ergogênica e potenciais riscos para a saúde. Revista Brasileira de Medicina do Esporte. Vol. 9. 2003. p. 43-56.

TIÊ PARMA YAMATO, BRUNO TIROTTI SARAGIOTTO, ALEXANDRE DIAS LOPES; prevalência de dor musculoesquelética em corredores de rua no momento em que precede o início da corrida, Rev. Bras. Ciênc. Esporte, Florianópolis, v. 33, n. 2, p. 475-482, abr./jun. 2011.

TRITSHLER, Kathleen. Medida e Avaliação em Educação Física e Esportes de Barrow \& McGee. Barueri, SP. MANOLE, 2003.

Wilmore, J. H.; Costill, D. L. Fisiologia do Esporte e do Exercício. São Paulo. Manole, 2a edição. 2001. 